\title{
Medicine in Old Age
}

\section{Drug Therapy in the Elderly}

\author{
M. R. P. HALL
}

British Medical fournal, 1973, 4, 582-584

\section{General Principles}

The efficiency of the individual organs and the body lessens with age. For instance, renal function diminishes, enzyme induction is delayed, cell membrane composition and total body salt and water contents are altered, and lean body mass is reduced. Consequently, the elderly differ from younger people in their response to drugs, for their ability to handle drugs by absorption, detoxication, and excretion is naturally effected. Not surprisingly, therefore, iatrogenic disease related to drugs is very common.

Fortunately, as a rule, absorption is less affected by age than excretion and detoxication. Hence the failure of an elderly person to respond to an effective drug is nearly always due to failure of the patient to take the drug rather than to absorb it. Large loading doses of drugs are, therefore, rarely necessary and in most cases it is preferable to start with a small dose, particularly if the drug is potentially toxic and likely to be poorly excreted. This does not mean that large doses of some drugs should not be given to the elderly if indicated-for example, antibiotics in acute infections or diuretics in the early stages of heart failure. Nevertheless, in using drugs in the elderly it is wise to observe a few simple rules:

(1) Know the pharmacological action of the drug being used and in particular how it is metabolized and excreted.

(2) Use the lowest dose that is effective in the individual patient. Higher blood concentrations per dose are achieved and the half life of some drugs-for example, cortisol or digoxin-is prolonged in the elderly, and this can lead to acoumulation and the need for lower and less frequent dosage. Drug dose should, therefore, be titrated with patient response.

(3) Use the fewest drugs the patient needs. Memory, particularly short-term memory, deteriorates with age; consequently complex drug regimens may be mismanaged.

(4) Do not use drugs to treat symptoms without first discovering the cause of the symptoms. These may sometimes be due to a social deprivation syndrome which may be better dealt with by a trained social worker.

(5) Do not withhold drugs on account of old age particularly when drug therapy may improve the old person's quality of life.

(6) Do not use a drug if the symptoms it causes are worse than those it is supposed to relieve. Hence knowledge of a drug's side effects is important and one should remember that sometimes adverse reactions may be individual to the

Faculty of Medicine, Southampton General Hospital, Tremona Road, Southampton SO9 4XY

M. R. P. HALL, B.M., F.R.C.P., (Lond. Ed.), Professor of Geriatric Medicine

patient and may occur to a drug which has been taken successfully for many years.

(7) Do not continue to use a drug if it is no longer necessary. It is a wise rule to review repeat prescriptions quarterly in all elderly patients.

\section{Drugs Acting on the Cardiovascular System}

Heart failure is common in old age and digitalis glycosides remain the key to successful treatment. Their poor tolerance and adverse effects have been known for years. Possibly the use of the pure glycoside rather than a preparation of crude digitalis leaf and modern manufacturing techniques may have contributed to this intolerance. Ewy et al. ${ }^{1}$ found that in the elderly the same dose of digoxin produced higher blood levels and longer blood half life. Rules (1) and (2) apply, and great care must be taken in the administration of all digitalis derivatives, the dose always being titrated against patient response. A dose of $0.25 \mathrm{mg}$ daily is usually effective, though some individuals need less, in which case the small paediatric/geriatric (PG) tablets of $0.0625 \mathrm{mg}$ strength may be useful. Maintenance therapy is probably unnecessary in about $70 \%$ of cases ${ }^{2}$ and the drug may be withdrawn in many instances quite safely.

That potassium depletion causes digitalis intolerance is well reoognized. It may be produced not only by the use of powerful diuretics but may already exist as a result of reduced intake owing to poor diet. The serum potassium may be little guide to the total body potassium content, though low serum levels are nearly always associated with low total body levels. ${ }^{3}$

Negative potassium balance in congestive heart failure may be high, and large potassium supplements are necessary when treating acute heart failure, panticularly if diuretics are also used-for example, a minimum of $48 \mathrm{mEq} / 24 \mathrm{hr}$. Nevertheless, since impairment of renal function occurs with age, it may not be necessary for large doses to be given for long, and potassium levels should be closely monitored, the blood levels being checked at weekly intervals until the heart failure is controlled. Once heart failure is controlled, contimued maintenance therapy with potassium salts may be unnecessary. ${ }^{4}$ If potassium supplements are discontinued the patient must be watched for signs of hypokalaemia (oonfusion, lassitude, anorexia, cardiac arrhythmias, and muscular weakness) and blood levels checked at regular intervals, particularly if diuretics are continued.

\section{DIURETICS}

Diuretics are commonly used in old people to treat heart failure and oedema. Indeed, they are probably over-used to treat dependent oedema, which is unlikely to respond, so that they produce sodium and potassium depletion, postural 
hypotension, immobility, constipation, faecal impaction with double incontinence, and a state of misery and social inacceptability. The actual drug used is a matter of personal choice, since most are effective-but probably bendrofluazide, chlorthalidone, and frusemide are the most useful. The dose should be large enough to be effective and very large doses-for example, frusemide $240 \mathrm{mg}$ - may be necessary on occasions. It should be remembered that all diuretics may provoke gout and carbohydrate intolerance. Aldosterone antagonists such as spironolactone may potentiate their action and prevent excessive potassium depletion. Dall et al. ${ }^{5}$ have reported that ameloride in combination with hydrochlorothiazide may conserve potassium. The drug, however, should be used with caution in the elderly since it may give rise to high blood potassium levels.

\section{BETA-BLOCKERS}

The adrenergic B-receptor blockers may be used to control supraventricular arrhythmias in elderly patients. Practolol is probably the most useful since it is less likely to produce bradycardia, heart failure, and bronchospasm than propranolol. These drugs may be particularly useful in controlling the tachycardia associated with hyperthynoidism.

Hypotensive drugs should be used with caution in the elderly, and some physicians in geriatric medicine question their use at all. Nevertheless, rule (5) applies to this group of drugs and if their use enables the patient to lead a more pleasant and active life they should be used. The liability of some preparations to produce mental depression must be recognized.

The vasodilator drugs will increase peripheral circulation in the limbs as well as the cerebral circulation. Their use, therefore, to improve both the symptoms of peripheral vascular disease and the effects of cerebrovascular disease is advocated by their makers. Improvement in the efficiency of blood flow through diseased vessels, however, is doubtful and consequently their efficacy in improving symptoms. Nevertheless, some preparations do alter the metabolism of cells in the brain, so that some effect is a theoretical possibility. As a group of drugs they seem to produce little in the way of side effects even if their value remains unproved.

\section{DRUGS ACTING ON THE CENTRAL NERVOUS SYSTEM}

The elderly commonly suffer from agitation, restlessness, and insomnia. In a busy clinic it is often difficult to elucidate the cause of these symptoms and hence drugs are frequently prescribed. Rule (4) panticularly applies to these, for they are all potentially dangerous to the elderly. Nevertheless, they may be effective in relieving symptoms and hence useful. Some old people are extremely sensitive to some of these drugs: even quite small doses may produce soporific states, and hence small doses should be used initially.

Probably the most useful hypnotics are one of the modern chloral derivatives such as dichloralphenazone (Welldorm) or triclofos (Tricloryl). Alternatives to these are glutethimide (Doriden), which may give rise to cerebellar signs and vitamin D deficiency in some old people; chlormethiazole (Heminevrin); nitrazepam (Mogadon); and meprobamate (Miltown, Idemin, Equanil, Pathibamate, Mepavion). Barbiturates are not recommended, for the elderly tolerate them poorly and they may give rise to lethargy, depression, and confusion.

\section{SEDATIVES AND TRANQUILIZERS}

Of the sedatives and tranquilizers, the most widely used are diazepam (Valium) and chlordiazepoxide (Librium). Both drugs, however, may provoke a feeling of weakness in some old people-and patients who have had cerebrovascular accidents are particularly susceptible. Undoubtedly agitation and hallucinations are most easily controlled by the phenothiazine group. Despite its tendency to give rise to jaundice in some people, chlorpromazine (Largactil) is probably the most effective and widely used. Doses should be kept as small as possible. It is wise to start with a dose of $10 \mathrm{mg}$, increasing this as necessary. Alternatives are promazine (Sparine), which is weaker, and thioridazine (Melleril), which may be more effective in some patients. More potent drugs in this group are trifluoperazine (Stelazine) and haloperidol (Serenace); the latter, which comes in the form of collourless and tasteless drops, may be particularly useful in controlling the very agitated patient. The usual dose is $0.5 \mathrm{mg}$ to $1.5 \mathrm{mg}$ three times a day. Similarly the decanoate and enanthate forms of fluphenazine may be useful, since a single injection will control symptoms for quite long periods. It should be remembered that all these drugs produce side effects, and in particular features of Parkinsonism, so that it may be necessary to give an antiparkinsonian preparation simultaneously. They should also not be used in patients who are vulnerable to accidental hypothermia, and they may provoke postural hypotension.

The incidence of depressive illness rises with age and the treatment of patients with this condition should be supervised by a psychiatrist. Short courses of electro-shock therapy may often be more effective than long-continued drug taking. If drugs are used the tricyclic group of antidepressant drugs is most useful. These drugs may cause urinary retention, and constipation, and may precipitate or aggravate glaucoma. They may also provoke severe postural hypotension and aggravate dryness of the mouth-or even cause lingual or oral ulcers.

Rules (1) and (2) apply particularly to all these drugs but large doses should not be withheld from those patients who need them.

\section{PARKINSON'S DISEASE}

Treatment with L-dopa represents a considerable advance. It must, however, be used cautiously, for failure of treatment is often due to incorrect prescription. Thus the starting dose should not be greater than $125 \mathrm{mg}$ daily, and increments should be at weekly intervals, and of $125 \mathrm{mg}$ dose, until the daily dose equals $0.5 \mathrm{~g}$. Some elderly respond to amantadine hydrochloride in an initial dose of $100 \mathrm{mg}$ daily, which is increased to $100 \mathrm{mg}$ twice daily after one week. Severe nausea and vomiting are common adverse reactions to L-dopa and may be due to peaking of blood levels, and a slow-release preparation prevents this. Consequently Brocadopa Temtabs may be the most appropriate L-dopa preparation for elderly patients. Optimum dosage may be lower than in younger people and improvement may continue for months or even years. The older antiparkinsonian drugs may have a place in the treatment of those patients who cannot tolerate $\mathrm{L}$-dopa, but they should all be used with care, and rules (1) and (2) apply.

\section{Relief of Pain}

Pain and discomfont are common symptoms, being associated with skeletal disorders as well as with cancer. ${ }^{6-8}$ Undoubtedly it is much easier to prevent the onset of pain than to relieve it once it has occurred. Pain should not be allowed to ebb and flow; hence analgesics, sedatives, anti-inflammatory drugs, and narcotics should be given regularly and patients instructed in their proper use to prevent the development of pain and minimize the symptom as much as possible.

Soluble aspirin probably remains the most effective and 
useful analgesic, particularly in the treatment of skeletal as opposed to visceral pain. The possibility of gastrointestinal bleeding must be remembered; some preparations-for example, benorylate, safapryn, or Caprin-may be safer but are more expensive. Paracetamol, either alone or in combination with other analgesics, is safer but less effective, though its absorption and consequently action may be enhanced by sorbitol. The anti-inflammatory drugs phenlybutazone (Butazolidin), oxyphenbutazone (Tanderil), indomethacin (Indocid), and ibuprofen (Brufen) are effective in relieving pain but all tend to give rise to gastrointestinal symptoms-though ibuprofen seems better tolerated by most of the elderly. Narcotics are often necessary to treat severe pain. If given in conjunction with chlorpromazine they may be used in quite small doses-for example, pethidine 150$300 \mathrm{mg}$ daily-thus enabling the patient to remain alert yet be free from pain. In very severe pain diamorphine remains the drug of choice in combination with chlorpromazine.

Corticosteroids rarely need to be used and unless specifically indicated they should be avoided. They are sometimes useful for the treatment of arthralgia, which does not respond to other analgesics-for example, severe late-onset rheumatoid arthritis. Often prednisone, 1 or $2 \mathrm{mg}$ three times a day, is effective. Similar doses may also be used in the treatment of asthma. Larger doses are necessary in the treatment of temporal arteritis and polymyalgia rheumatica. Here the initial dose should be high-reduction, which should be achieved as rapidly as possible, being titrated against the erythrocyte sedimentation rate. All patients on corticosteroids should also be given an anabolic steroid concomitantly in an attempt to prevent osteoporosis. Otherwise the regular use of anabolic steroids is probably of unproved value.

\section{Treatment of Infection}

There are no special contraindications to the use of anti- bacterial drugs in the elderly. It should, however, be remembered that many are poorly eliminated; thus blood levels will be higher and toxic reactions may be more common. Antibiotic and antibacterial drugs should be given by mouth since injections may cause sterile abscesses and the resulting tissue breakdown may produce pressure sores. Concomitant monilial infections may occur in severely debilitated patients.

\section{Conclusions}

It is impossible in a short article such as this to cover all aspects of drug therapy in the elderly. For instance, replacement therapy using hormones such as thyroxine, or vitamins such as vitamin $B_{12}$ and folic acid, may be necessary. Vitamins, particularly C and D, and minerals, such as iron, may be indicated in the housebound and other elderly prone to subnutrition. While there are no special preparations which will combat old age, the elderly will benefit as much from appropriate drug therapy as any young person. The key to good drug therapy is accurate diagnosis, assessment of the objectives of treatment, and use of the appropriate drug or drugs, bearing in mind the rules already outlined, which should govern the use of any drug. Good therapy is not easy but it is rewarding.

\author{
References \\ 1 Ewy, A., Kapadia, G., Yao, L., Lullin, M., and Marcus, F. I., Circulation,
1969, 39, 449. \\ 2 Dall, J. L. C., British Medical fournal, 1970, 2, 706. \\ ${ }^{3}$ Cox, J. R., Pearson, R. E., and Speight, C. J., Gerontologia Clinica, 1972, \\ 13,233 . \\ 13 Down, P. F., Polak, A., Rao, R., and Mead, J. A., Lancet, 1972, 2, 721. \\ 5 Dall, J. L. C., MacFarland, J. P. R., and Kennedy, R. D., Proceedings \\ of the VIth European Congress of Clinical Gerontology, p. 371-373, \\ 6 Sinclair, D., British fournal of Hospital Medicine, 1973, 9, 568. \\ 7 Merskey, H., British fournal of Hospital Medicine, 1973, 9, 574 \\ ${ }^{8}$ Lipton, S., British fournal of Hospital Medicine, 1973, 9, 583.
}

\section{Any Questions?}

\section{We publish below a selection of questions and answers of general interest}

\section{Regular Bedtime Use of Diphenhydramine Hydrochloride}

Is there any possibility of side effects or harm of any kind resulting from the regular bedtime use of $25-75 \mathrm{mg}$ of Benadryl?

Antihistamine drugs such as diphenhydramine hydrochloride (Benadryl) are remarkably free of side effects. The main problem is usually drowsiness, which in the present circumstances would not be a problem except that the drug has a fairly long action and drowsiness and impairment of concentration might be carried over to the next day. Other side effects-nausea, vomiting, headache, dryness of the mouth, and disturbances of micturition-are uncommon. Leucopenia and agranulocytosis are very rare in treatment and if the patient has already been on the drug for some time it is unlikely that these will present a problem.

The real question is whether it is desirable that any patient should be on diphenhydramine regularly at bed-time. If the drug is being used as a hypnotic there are better drugs for this and it is seldom necessary for the drug to be taken regularly for its antihistamine action at this time. The real dangers of any regular medication are two-fold. Firstly, the patient becomes habituated to the drug and is then taking it without obtaining any continuing therapeutic benefit. Secondly, both the patient and the doctor are liable to forget the fact that the drug is being taken and when a fresh medical problem arises other drugs may be prescribed which will interact with the regular medication with serious, even dangerous results. In the case of diphenhydramine this might arise if sedative, tranquillizing, atropine-like substances were taken by the patient.

\section{Gold Treatment for Spondylitis of the Neck}

Is there any evidence that gold salts are of help in the treatment of spondylitis of the neck? Are there any recent advances in therapy for this chronic condition?

Rheumatoid arthritis of the neck improves on gold therapy as do other joints in this disorder but gold salts have little effect on other conditions and are not given in ankylosing spondylitis or cervical spondylosis. The treatment of spondylitis depends entirely on the cause of the spondylitis for many different disorders may manifest in this way. 